STRUCTURAL BIOLOGY COMMUNICATIONS

ISSN 2053-230X

\section{Crystal structures of the ligand-binding domain of human peroxisome proliferator-activated receptor $\delta$ in complexes with phenylpropanoic acid derivatives and a pyridine carboxylic acid derivative}

\author{
Takuji Oyama, $^{\text {a* Kazuki Takiguchi }}{ }^{\mathrm{a}}$ and Hiroyuki Miyachi ${ }^{\mathrm{b}}$
}

Received 29 November 2021

Accepted 12 January 2022

Edited by A. Nakagawa, Osaka University, Japan

Keywords: nuclear receptors; peroxisome proliferator-activated receptor $\delta$; PPAR $\delta$; ligand-binding domain.

PDB references: PPAR $\delta$ LBD-JK122, 7vwe; PPAR $\delta$ LBD-TIPP-204, 7vwf; PPAR $\delta$ LBDJKPL38, 7vwg; PPAR $\delta$ LBD-JKPL39, 7vwh

Supporting information: this article has supporting information at journals.iucr.org/f

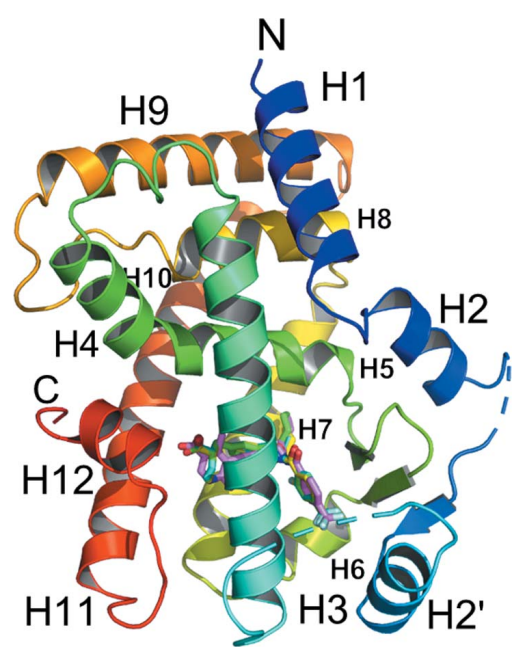

${ }^{\mathbf{a}}$ Department of Biotechnology, Faculty of Life and Environmental Sciences, University of Yamanashi, Japan, and ${ }^{\mathbf{b}}$ Lead Exploration Unit, Drug Discovery Initiative, The University of Tokyo, Japan. *Correspondence e-mail: takujio@yamanashi.ac.jp

Peroxisome proliferator-activated receptor $\delta(\operatorname{PPAR} \delta)$ is a member of the nuclear receptor family and regulates glucose and lipid homeostasis in a liganddependent manner. Numerous phenylpropanoic acid derivatives targeting three PPAR subtypes (PPAR $\alpha, \operatorname{PPAR} \gamma$ and $\operatorname{PPAR} \delta$ ) have been developed towards the treatment of serious diseases such as lipid-metabolism disorders. In spite of the increasing attraction of $\operatorname{PPAR} \delta$ as a pharmaceutical target, only a limited number of protein-ligand complex structures are available. Here, four crystal structures of the ligand-binding domain of $\operatorname{PPAR} \delta$ in complexes with phenylpropanoic acid derivatives and a pyridine carboxylic acid derivative are described, including an updated, higher resolution version of a previous studied structure and three novel structures. These structures showed that the ligands were bound in the ligand-binding pocket of the receptor in a similar manner but with minor variations. The results could provide variable structural information for the further design and development of ligands targeting PPAR $\delta$.

\section{Introduction}

Nuclear receptors are transcription factors that are activated in a ligand-dependent manner (Georgiadi \& Kersten, 2012; Dubois et al., 2017). Among them, peroxisome proliferatoractivated receptors (PPARs) consist of three subtypes: $\operatorname{PPAR} \alpha, \operatorname{PPAR} \gamma$ and $\operatorname{PPAR} \delta$. They are involved in glucose and lipid homeostasis despite their different pharmacological properties and tissue distributions. Upon binding of agonists, PPARs adopt the activated conformation to interact with coactivator proteins, and then further bind the target DNA sequences as heterodimers with the retinoid $\mathrm{X}$ receptor (Fig. 1a; Chandra et al., 2008). $\operatorname{PPAR} \alpha$ and PPAR $\gamma$ have long attracted attention as targets for the development of therapeutic agents for hyperlipidemia and diabetes, respectively (Mirza et al., 2019). PPAR $\delta$ has not received as much attention as the other two subtypes, probably due to its wide distribution in the body. However, recent studies have indicated that $\operatorname{PPAR} \delta$ is involved in various diseases such as a variety of cancers (Müller, 2017), non-alcoholic fatty liver disease (NAFLD; Zarei et al., 2021) and neuroinflammation (Strosznajder et al., 2021). Therefore, $\operatorname{PPAR} \delta$ has recently gained attention as a target for therapeutic drug development. Since the first structural report of the ligand-binding domain of $\operatorname{PPAR} \delta(\operatorname{PPAR} \delta$ LBD) in complex with intrinsic fatty acids and with a synthetic ligand (Xu et al., 1999), numerous protein-ligand complex structures have been reported. 
Recently, Wu et al. (2017) reported 17 crystal structures of PPAR $\delta$ LBD-ligand complexes. Nonetheless, the number of PPAR $\delta$ LBD-ligand complex structures deposited in the Protein Data Bank (PDB) is still limited to 43 (as of 17 November 2021), which is fewer than those of PPAR $\alpha$ (60) and PPAR $\gamma$ (254).

We have developed a series of phenylpropanoic acid derivative compounds targeting the three PPAR subtypes based on structure-activity relationship (SAR) studies, using the PPAR $\alpha$-specific compound KCL as a lead compound (Fig. $1 b$; Miyachi, 2021). When we view the ligand-binding pockets of PPARs as Y-shaped with three arms (arms 1, 2 and 3), these compounds are bound so that the head, branch and tail portions fit into each arm (Oyama et al., 2009, 2021). PPAR $\delta$ specific agonists possess a longer alkoxy residue (typically butoxy to $n$-hexyloxy) at the branch position, while PPAR $\alpha / \delta$ dual agonism is exhibited when the alkoxy part is replaced with a shorter part (methoxy to propoxy) (Kasuga et al., 2007). We determined crystal structures of PPAR $\delta$ LBD in complex with TIPP-401 (an $\alpha / \delta$ dual agonist) and TIPP-204 ( $\delta$-specific; $\left.\mathrm{EC}_{50}=1.9 \mathrm{nM}\right)($ Oyama et al., 2009), and further developed other derivatives such as JKPL38, which is an $\alpha / \delta$ dual agonist with a propoxy residue $\left(\mathrm{EC}_{50}=6.8 \mathrm{n} M\right)$, and JKPL39, a $\delta$ specific agonist $\left(\mathrm{EC}_{50}=45 \mathrm{n} M\right)$ which has a longer $n$-hexyloxy portion at the branch (Fig. 1b; Kasuga, Ishida et al., 2009).

We also developed biphenyl carboxylic acid derivatives as $\operatorname{PPAR} \delta$ partial agonists and antagonists (Kasuga, Oyama et al., 2009). Furthermore, in an attempt to improve the watersolubility of these compounds, we obtained the water-soluble $\operatorname{PPAR} \delta$-specific partial agonist JK122 $\left(\mathrm{EC}_{50}=76 \mathrm{n} M\right)$ with a dimethylpyridine carboxylic acid as the head group (Kasuga et al., 2010). However, their binding mode to PPAR $\delta$ LBD has not been examined experimentally, which has partly limited the SAR study cycle to obtain better ligands.

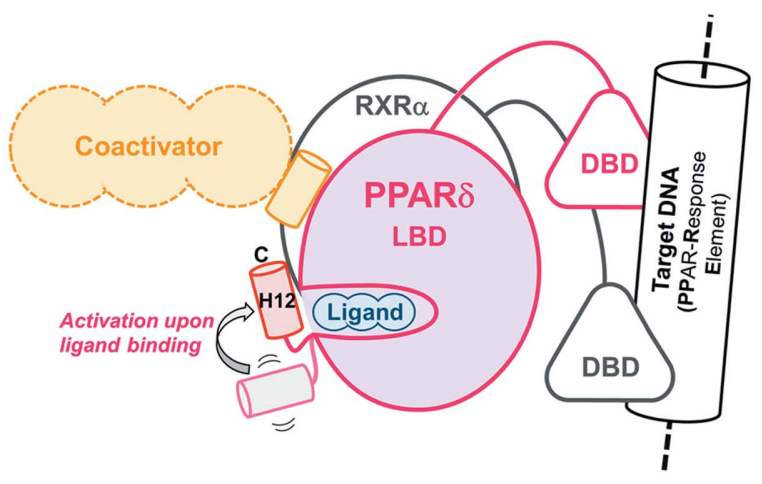

(a)

PPAR $\alpha$-specific

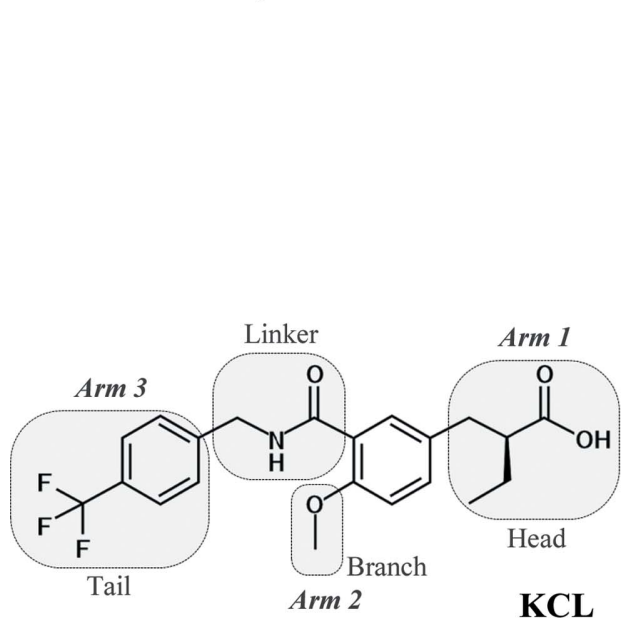

PPAR $\alpha / \delta$-specific

\section{$\operatorname{PPAR} \delta$-specific}

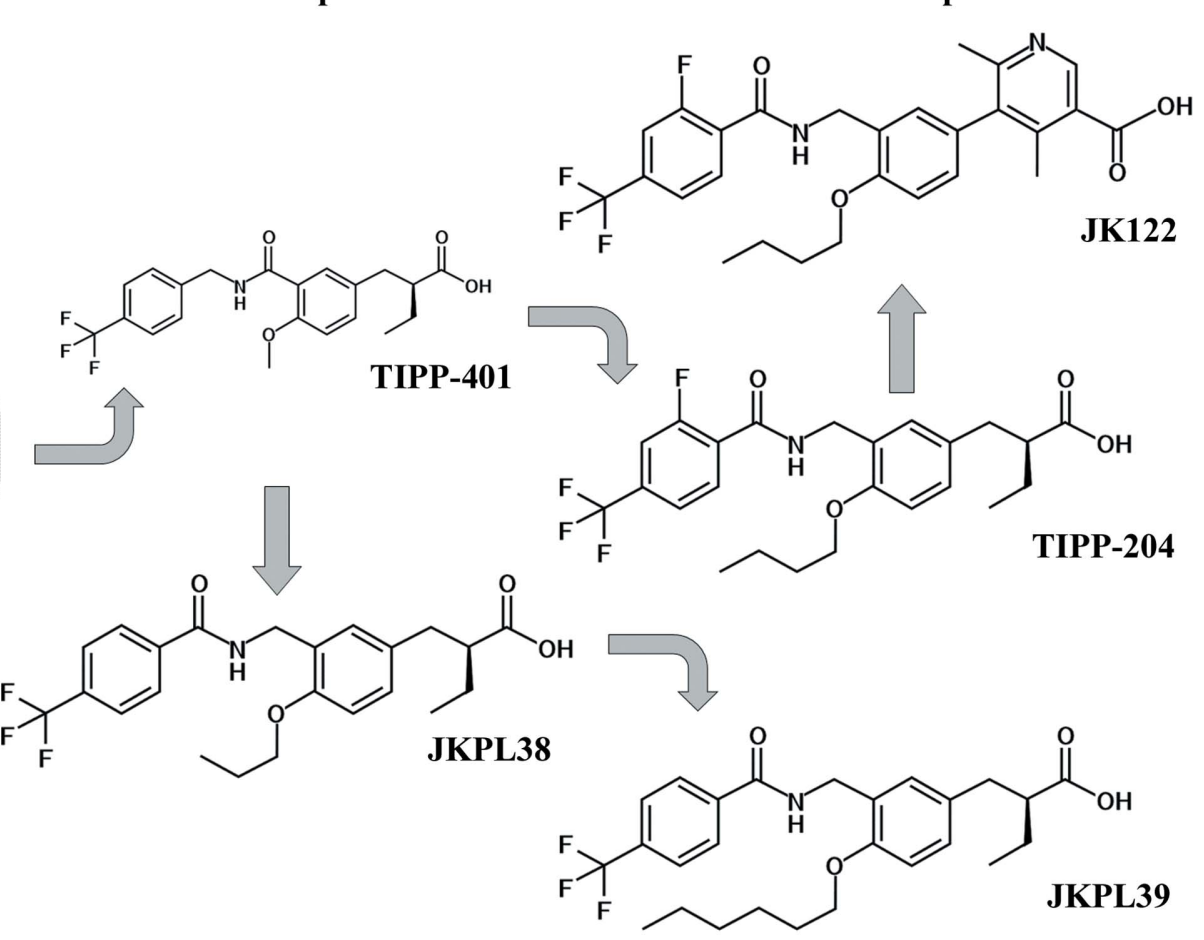

Figure 1

(b)

(a) A schematic diagram of the ligand-activated $\operatorname{PPAR} \delta-\mathrm{RXR} \alpha$ heterodimer. (b) Chemical structures of the ligands used in this study. Functional units are shown on the KCL structure with the binding arms in the ligand-binding pocket of PPAR $\delta$. 
In this study, we have slightly modified the expression protocol of PPAR $\delta$ LBD in Escherichia coli and thereby succeeded in the crystallization and structure determination of four protein-ligand complexes. We previously reported a crystal structure of PPAR $\delta$ LBD-TIPP-204 at $3.0 \AA$ resolution. Here, we obtained a higher resolution (1.9 $\AA$ ) crystal of this complex, which updated the binding mode of TIPP-204. We also determined three novel structures of PPAR $\delta$ LBD complexed with JKPL38, JKPL39 and JK122. These structures could provide useful information for the further development of better ligands for $\operatorname{PPAR} \delta$.

\section{Materials and methods}

\subsection{Macromolecule production}

The protein expression (Table 1) and purification of human $\operatorname{PPAR} \delta$ LBD were performed using almost the same procedure as described previously (Oyama et al., 2009), except for the use of a different E. coli expression cell strain: ArcticExpress (DE3) (Agilent Technology). This strain expresses two chaperone proteins derived from a microorganism in the Antarctic Ocean when exposed to a low temperature of around $286 \mathrm{~K}$. The cells were transformed using the pET-28a vector, into which the $\operatorname{PPAR} \delta \mathrm{LBD}$ gene was inserted, and were cultured in Terrific Broth at $310 \mathrm{~K}$ until the optical density $\left(\mathrm{OD}_{600}\right)$ reached $0.6-0.8$. The cells were rapidly cooled to $286 \mathrm{~K}$ using a bucket of ice water, and isopropyl $\beta$-D-1thiogalactopyranoside (IPTG) was then added to the culture medium to a final concentration of $1 \mathrm{~m} M$ for the expression of $\operatorname{PPAR} \delta$ LBD. The cells were further incubated at $286 \mathrm{~K}$ for $48 \mathrm{~h}$ and harvested by centrifugation $(3752 \mathrm{~g}, 10 \mathrm{~min}$ at $277 \mathrm{~K}$ ). Protein purification using three-step column chromatography (a nickel-chelating column followed by cation-exchange and gel-filtration columns) was conducted as described previously. The purified protein was concentrated to $7 \mathrm{mg} \mathrm{ml}^{-1}$ using Amicon Ultra concentrators (molecular-weight cutoff 3000). To form protein-ligand complexes, an aliquot of a concentrated solution of each ligand in $100 \%$ dimethyl sulfoxide (DMSO) was added to the protein solution at a protein:ligand molar ratio of 1:5 and was then used for crystallization.

\subsection{Crystallization}

Complexes of PPAR $\delta$ LBD with TIPP-204, JKPL38 and JKPL39 were successfully crystallized by the hanging-drop vapor-diffusion method using the previously reported crystallization reservoir solution (reservoir $A$ in Table 2; Oyama et al., 2009). Plate-like crystals (with average dimensions of approximately $50 \times 50 \times 25 \mu \mathrm{m}$ ) were obtained within several days. The crystals were cryo-harvested using reservoir solution supplemented with 20\%(w/v) PEG 1000 and flash-cooled in liquid nitrogen. For crystallization of the complex with JK122, crystallization screening was performed using a Mosquito semi-automatic dispensing robot (TTP Labtech) with the commercially available screening kits JCSG Core Suites I-IV (NeXtal). Diffraction-quality crystals were obtained by the hanging-drop vapor-diffusion method, in which the protein-
Table 1

Macromolecule-production information.

\begin{tabular}{|c|c|}
\hline Source organism & Homo sapiens \\
\hline Expression vector & pET-28a \\
\hline Expression host & Escherichia coli BL21 (DE3) \\
\hline \multirow{10}{*}{$\begin{array}{l}\text { Complete amino-acid sequence } \\
\text { of the construct produced }\end{array}$} & GSHMPQVADLKAFSKHIYNAYLKNFNMTKK \\
\hline & KARS ILTGKASHTAPFVIHDIETLWQAE \\
\hline & KGLVWKQLVNGLPPYKEISVHVFYRCQC \\
\hline & TTVETVRELTEFAKS I PSFSSLFLNDQV \\
\hline & TLLKYGVHEAI FAMLAS IVNKDGLLVAN \\
\hline & GSGFVTREFLRSLRKPFSDI IEPKFEFA \\
\hline & VKFNALELDDSDLALFIAAIILCGDRPG \\
\hline & LMNVPRVEAIQDTI LRALEFHLQANHPD \\
\hline & AQYLFPKLLQKMADLRQLVTEHAQMMQR \\
\hline & IKKTETETSLHPLLQEIYKDMY \\
\hline
\end{tabular}

Table 2

Crystallization.

\begin{tabular}{ll}
\hline Method & Hanging-drop vapor diffusion \\
Plate type & VDX plate \\
Temperature $(\mathrm{K})$ & 293 \\
Protein concentration $\left(\mathrm{mg} \mathrm{ml}^{-1}\right)$ & 7 \\
Buffer composition of protein & $20 \mathrm{~m} M$ HEPES, $10 \mathrm{~m} M$ DTT, $500 \mathrm{~m} M$ \\
$\quad$ solution & ammonium acetate pH 7.5 \\
Composition of reservoir & $A: 11-14 \%(w / v)$ PEG $4000,200 \mathrm{~m} M \mathrm{KCl}$, \\
$\quad$ solution & $40 \mathrm{~m} M$ bis-Tris methane, $6 \%(v / v)$ \\
& 1,3 -propanediol, $0.5 \%(w / v) n$-heptyl- \\
& $\beta$-D-glucopyranoside, $1 \mathrm{~m} M$ EDTA, \\
& $1 \mathrm{~m} M$ CaCl \\
& $B: 0.2 M$ potassium thiocyanate, $20 \%(w / v)$ \\
& PEG 3350. \\
Volume and ratio of drop & $1 \mu \mathrm{l}$ protein and $1 \mu$ reservoir solution \\
Volume of reservoir $(\mu \mathrm{l})$ & 500 \\
\hline
\end{tabular}

ligand complex solution was mixed with a reservoir solution consisting of $0.2 \mathrm{M}$ potassium thiocyanate, 20\%(w/v) PEG 3350 (JCSG Core I Suite condition C9) in a 1:1 ratio (reservoir $B$ in Table 2). Plate-like crystals similar to those of the abovementioned complexes were obtained within several days and were cryo-harvested using reservoir solution supplemented with $20 \%(w / v)$ glycerol.

\subsection{Data collection and processing}

X-ray diffraction data were collected on the AR-NE3A beamline at the Photon Factory (PF), Tsukuba, Japan. Diffraction images were recorded on an ADSC Quantum 270 CCD detector for the PPAR $\delta$ LBD-JK122 crystals or a Dectris PILATUS 2M-F pixel-array detector for the other crystals. Data were processed with XDS (Kabsch, 2010) and AIMLESS (Evans \& Murshudov, 2013). Statistics of X-ray diffraction data collection and processing are summarized in Table 3.

\subsection{Structure solution and refinement}

The crystal structures were solved by Phaser in the Phenix suite (Liebschner et al., 2019) using a previously determined PPAR $\delta$ LBD-ligand complex structure (PDB entry 2znq; Oyama et al., 2009) as a probe. Structure refinement was then performed by iterations of manual model rebuilding in Coot (Emsley et al., 2010) and crystallographic refinement using phenix.refine in Phenix. Statistics of structure refinement are summarized in Table 4. 
Table 3

Data collection and processing.

Values in parentheses are for the outer shell.

\begin{tabular}{|c|c|c|c|c|}
\hline Complex & $\operatorname{PPAR} \delta$ LBD-JK122 & PPAR $\delta$ LBD-TIPP-204 & PPAR $\delta$ LBD-JKPL38 & PPAR $\delta$ LBD-JKPL39 \\
\hline Diffraction source & AR-NE3A, PF & AR-NE3A, PF & AR-NE3A, PF & AR-NE3A, PF \\
\hline Wavelength $(\AA)$ & 1.00000 & 1.00000 & 1.00000 & 1.00000 \\
\hline Temperature (K) & 100 & 100 & 100 & 100 \\
\hline Detector & ADSC Quantum 270 & Dectris PILATUS 2M-F & Dectris PILATUS 2M-F & Dectris PILATUS $2 \mathrm{M}-\mathrm{F}$ \\
\hline Space group & $P 2_{1}$ & $P 2_{1}$ & $P 2_{1}$ & $P 2_{1}$ \\
\hline$a, b, c(\AA)$ & $39.12,92.30,96.09$ & $39.56,94.25,96.31$ & $39.50,94.31,96.25$ & $39.59,94.14,96.18$ \\
\hline$\alpha, \beta, \gamma\left({ }^{\circ}\right)$ & $90,98.06,90$ & $90,97.32,90$ & $90,97.37,90$ & $90,96.64,90$ \\
\hline Mosaicity $\left({ }^{\circ}\right)$ & 0.21 & 0.11 & 0.15 & 0.16 \\
\hline Resolution range $(\AA)$ & $50.0-3.00(3.18-3.00)$ & $50.0-1.90(1.93-1.90)$ & $50.0-2.25(2.27-2.20)$ & $50.0-2.10(2.16-2.10)$ \\
\hline Total No. of reflections & $51094(8195)$ & $187262(11806)$ & 119135 (9950) & 134437 (11149) \\
\hline No. of unique reflections & $13610(2163)$ & $55080(3528)$ & $35214(3011)$ & $40370(3313)$ \\
\hline Completeness (\%) & $99.9(99.9)$ & $99.8(99.9)$ & $99.1(99.9)$ & $98.7(99.7)$ \\
\hline Multiplicity & $3.7(3.7)$ & $3.3(3.3)$ & $3.4(3.3)$ & $3.3(3.4)$ \\
\hline$\langle I / \sigma(I)\rangle$ & $7.9(2.0)$ & $12.8(1.9)$ & $12.8(3.1)$ & $14.3(3.0)$ \\
\hline$R_{\text {meas }}$ & $0.117(0.561)$ & $0.044(0.529)$ & $0.073(0.511)$ & $0.062(0.513)$ \\
\hline Overall $B$ factor from Wilson plot $\left(\AA^{2}\right)$ & 51.0 & 28.1 & 28.8 & 33.1 \\
\hline
\end{tabular}

Table 4

Structure refinement.

Values in parentheses are for the outer shell.

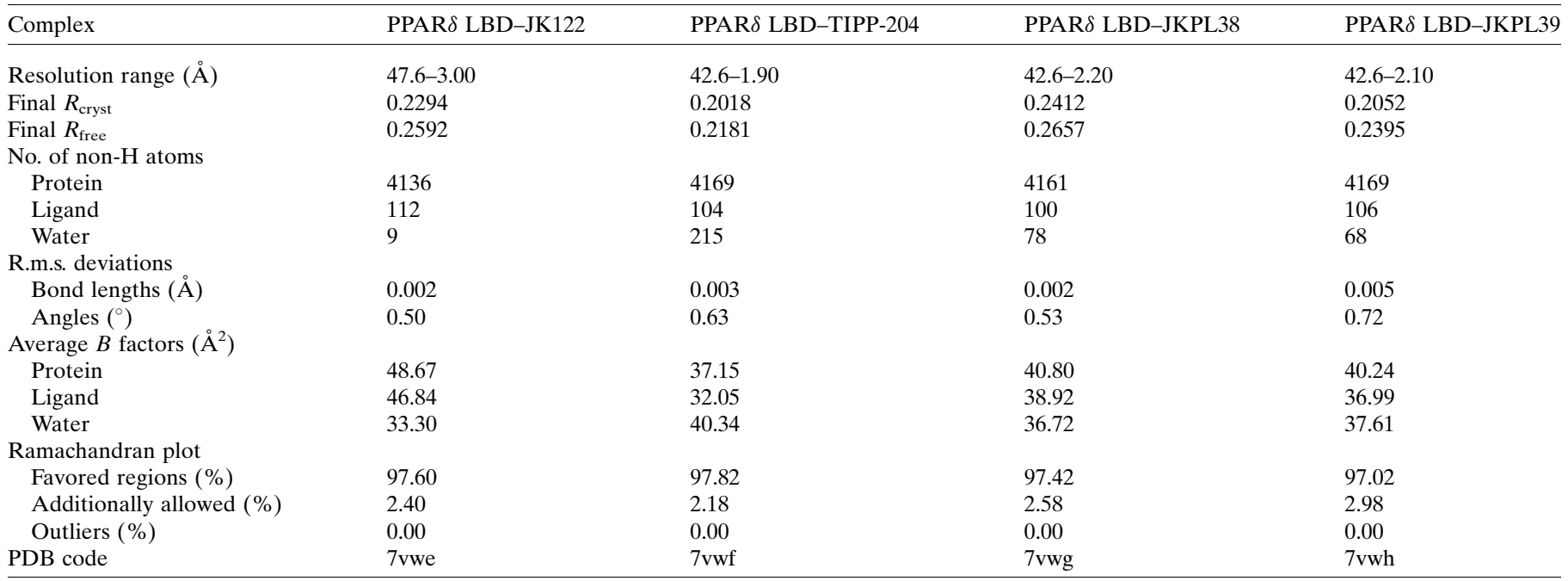

\section{Results and discussion}

\subsection{Updated structure of the PPAR $\delta$ LBD-TIPP-204 complex}

As in our previous study, all of the crystals belonged to space group $P 2_{1}$, with two complex molecules in the asymmetric unit which are related by noncrystallographic twofold symmetry (Fig. 2a). Both of the molecules in the asymmetric unit adopt the typical active conformation, in which Tyr473 in the C-terminal $\alpha$-helix $\mathrm{H} 12$ generally forms a hydrogen bond to the carboxy group of the bound ligands in arm 1 to form the typical active conformation of H12, while in arms 2 and 3 the protein possesses hydrophobic pockets that interact with nonpolar parts of the ligands (Figs. $2 b-2 e$ ). We previously determined a $3.0 \AA$ resolution structure of PPAR $\delta$ LBDTIPP-204 (PDB entry 2znp; Oyama et al., 2009). In this study, the crystals of the same complex exhibited an improved resolution of $1.9 \AA$, which could be attributed to the modified protein-expression method, as protein purification and complex crystallization were performed under the same conditions. Indeed, the purification yield was increased to about $5 \mathrm{mg}$ from a 11 culture of $E$. coli cells, compared with around $1 \mathrm{mg}$ in the previous protein-expression protocol. Comparison of the present (PDB entry 7vwe) and previous (PDB entry 2znp) structures of the ligand molecules in the ligand-binding pocket revealed only minor differences of the conformations of the alkyl groups, the head ethyl moiety and the branch butoxy residues, while the binding mode was unchanged overall (Fig. $3 a$ ). The root-mean-square deviation (r.m.s.d.) for the corresponding $255 \mathrm{C}^{\alpha}$ atoms between the present and previous structures (calculated using the SSM superpose option of Coot) is $0.45 \AA$.

\subsection{Structures of the PPAR $\delta$ LBD-JKPL38 and PPAR $\delta$ LBD-JKPL39 complexes}

JKPL38, TIPP-204 and JKPL39 have successively longer $n$-alkoxy groups on their branches, which are accommodated in arm 2 of the ligand-binding pocket (Fig. 1). PPAR $\delta$ has a 

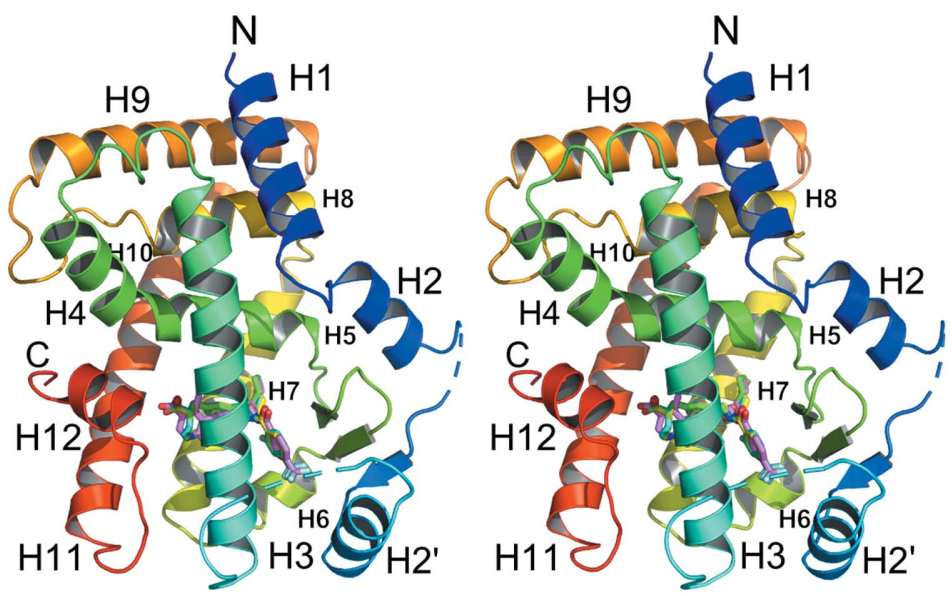

$(a)$

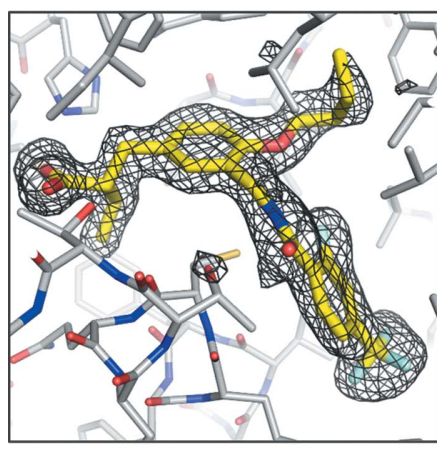

(b)

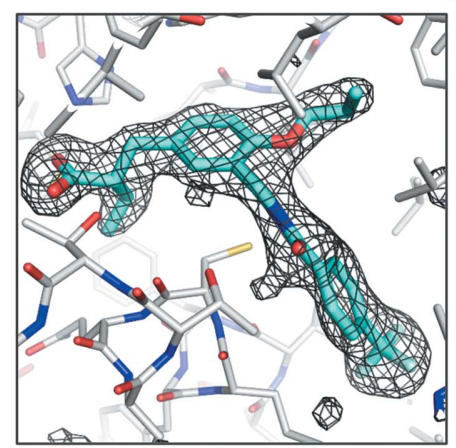

(c)

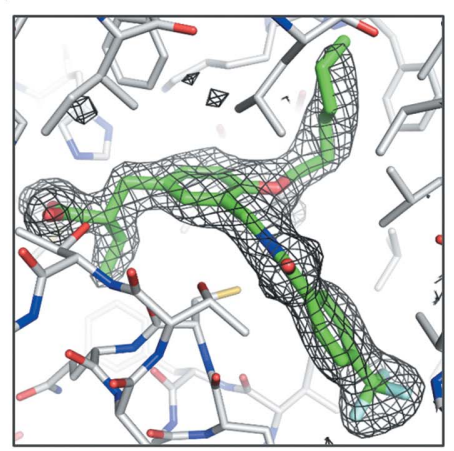

(d)

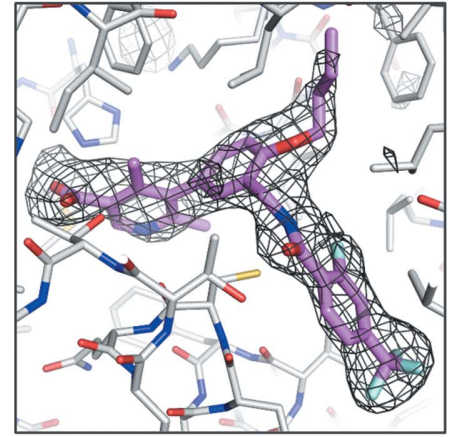

$(e)$

Figure 2

Structures of PPAR $\delta$ LBD-ligand complexes. (a) Overall view of the complexes. PPAR $\delta$ LDB is shown as a ribbon representation colored from blue (N-terminus) to red (C-terminus). Omit $F_{\mathrm{o}}-F_{\mathrm{c}}$ electron-density maps for (b) TIPP-204, (c) JKPL38, (d) JKPL39 and (e) JK122 are shown contoured at 3.0\%. Ligands are shown as stick models. C atoms of TIPP-204, JKPL38, JKPL39 and JK122 are colored yellow, cyan, green and violet, respectively. All atomic models were generated by PyMOL (http://www.pymol.org).

deeper arm 2 pocket than PPAR $\alpha$. Our previous studies have shown that compounds with methoxy to propoxy groups tend to function as dual PPAR $\alpha / \delta$ agonists, while those with longer groups than butoxy function as PPAR $\delta$-specific agonists. The conformations of the alkoxy groups of JKPL38, TIPP-204 and JKPL39 in the PPAR $\delta$ ligand-binding pocket were very similar to each other (Fig. $3 b$ ). There is no significant structural difference in the PPAR $\delta$ LBD structure (the r.m.s.d.s for 258 corresponding $\mathrm{C}^{\alpha}$ atoms are below $0.24 \AA$ ). A visual inspection implies that PPAR $\delta$ may bind ligands with an $n$-heptyloxy group at the branch.

\subsection{Structure of the PPAR $\delta$ LBD-JK122 complex}

The crystals of the PPAR $\delta$-JK122 complex obtained using the same crystallization conditions as used for the other ligand complexes were of poor quality. We then searched for crystallization conditions and obtained high-quality crystals under another condition. The crystals belonged to the same space group as the other crystals and had highly similar unit-cell parameters. JK122 is a water-soluble PPAR $\delta$-specific partial agonist developed on the basis of a biphenylcarboxylic acid compound and has a dimethylpyridine carboxylic acid unit at the head (Fig. 1; Kasuga et al., 2010). Consequently, the head of JK122 is slightly larger than the phenylpropaonic acid units of the other three ligands, and compared with them JK122 has one additional carbon in the connecting part between the head carboxy group and the linker benzene ring. Comparing the binding mode of JK122 in the ligand-binding pocket with that of TIPP-204 (the r.m.s.d. is $0.43 \AA$ for the superposition of 251 corresponding $\mathrm{C}^{\alpha}$ atoms), the head carboxy group forms a hydrogen bond to Tyr473 on $\mathrm{H} 12$ in a quite similar manner, including the location and direction. On the other hand, the rest of JK122 is pushed out towards arms 2 and 3 (by about $0.8 \AA$; Fig. $3 c$ ). It is currently unclear whether this slightly different mode of binding accounts for the difference in function between JK122 (a partial agonist) and TIPP-204 and the other ligands (full agonists).

\section{Concluding remarks}

We determined four crystal structures of PPAR $\delta$ LBD-ligand complexes, one of which is a high-resolution updated version, while the others are novel structures. A key to the success of this study was the increased stability of PPAR $\delta$ LBD due to its co-expression with the low-temperature-induced chaperone proteins in the host cells. The three phenylpropanoic acid derivatives bound to the ligand-binding pocket in a similar conformation, despite the variation in the alkoxy chain length in the branch portion. This was accomplished by the wide 


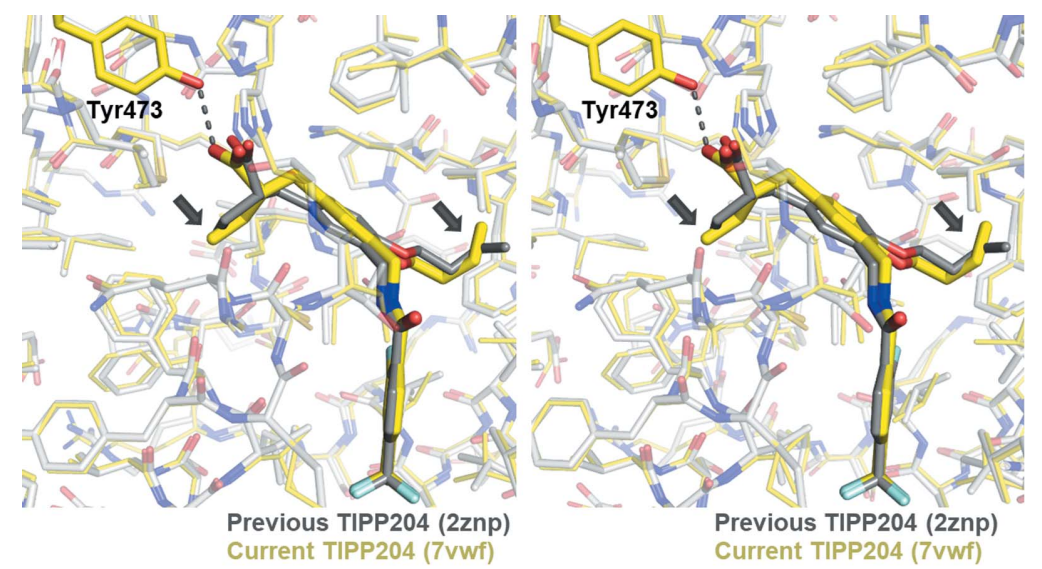

(a)

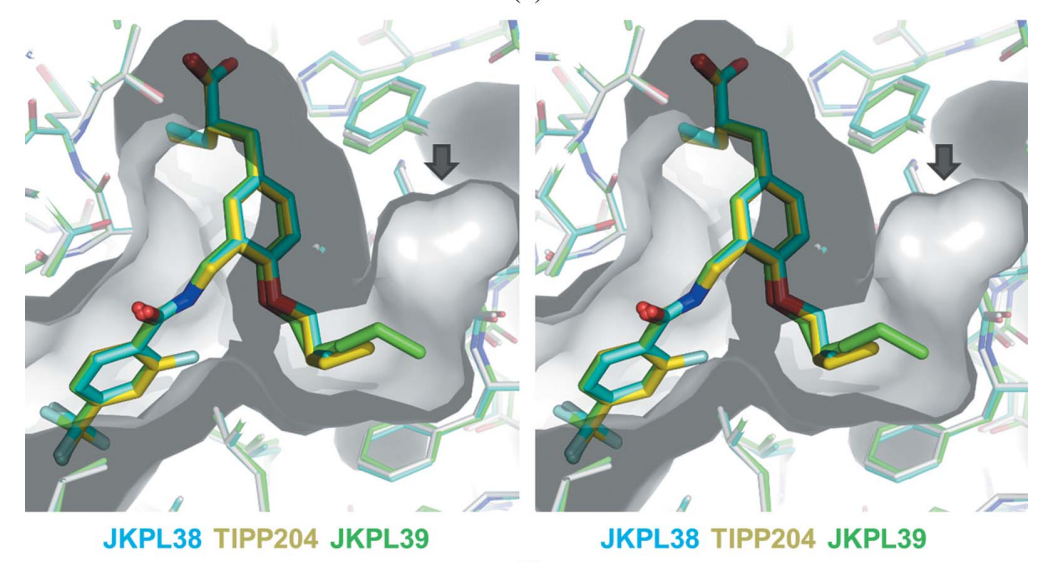

(b)

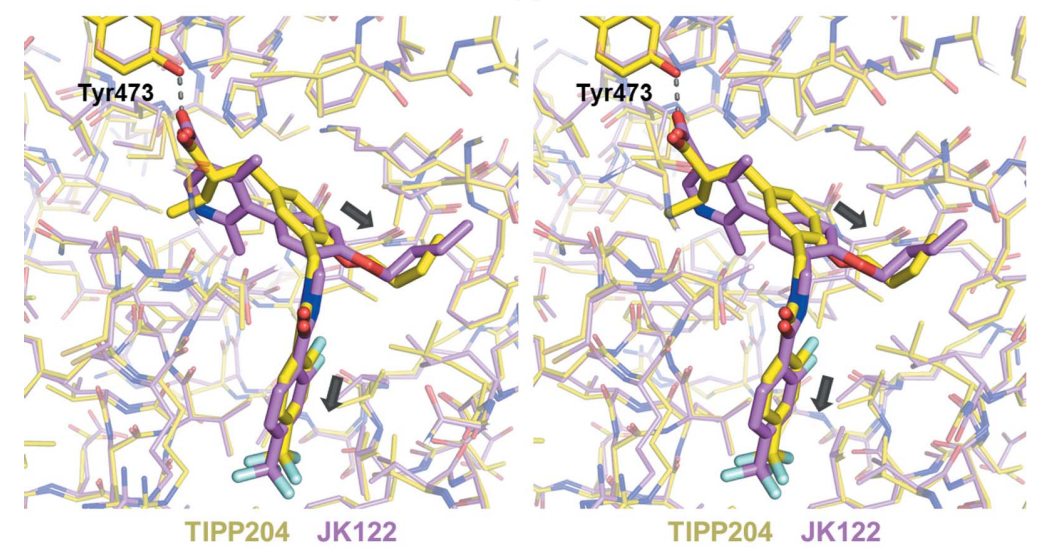

(c)

Figure 3

Ligand binding to PPAR $\delta$ LBD. (a) Structure of PPAR $\delta$ LBD-TIPP-204. A comparison is shown of the previous (gray) and current high-resolution (yellow) structures. Different conformations are indicated by arrows. (b) Superposition of PPAR $\delta$ LBD in complexes with TIPP-204 (yellow), JKPL38 (cyan) and JKPL39 (green). The arm 2 groove is represented by a transparent surface. An arrow indicates the bottom of arm 2. (c) Structure comparison of PPAR $\delta$ LBD-TIPP-204 (yellow) and PPAR $\delta$ LBD-JK122 (violet). The branch butoxy residue and the tail trifluoromethylbenzyl residue shift slightly (about $0.8 \AA$ ) towards arms 2 and 3, respectively, compared with the corresponding parts of TIPP-204 (indicated by arrows).

arm 2 groove that is characteristic of PPAR $\delta$. JK122 was located similarly to other ligands in the ligand-binding pocket around the head region in spite of its larger size, while the rest of the ligand shifted slightly towards arms 2 and 3. The previously developed biphenyl carboxylic acid derivatives (Kasuga, Oyama et al., 2009), which possess similar molecular structures to JK122, may bind to PPAR $\delta$ LBD in a similar manner to JK122. The present structures are expected to provide useful structural information for the future design and synthesis of novel compounds with improved characteristics.

\section{Acknowledgements}

X-ray diffraction experiments were performed at the Photon Factory with the approval of the Photon Factory Program Advisory Committee (Proposal Nos. 2011G598 and 
2015G024). We are grateful to the Photon Factory beamline staff for their assistance with X-ray diffraction data collection. We also thank Dr Jun-Ichi Kasuga and Ms Hitomi Nakamura for their help in sample preparation. Professor Masami Kusunoki is acknowledged for his encouragement during this study.

\section{Funding information}

Funding for this research was provided by: Japan Science and Technology Agency, Adaptable and Seamless Technology Transfer Program through Target-Driven R\&D (grant No. JPMJTM20NM to Takuji Oyama); Collaborative Research Program of Institute for Protein Research, Osaka University (grant No. CR-20-02 to Takuji Oyama).

\section{References}

Chandra, V., Huang, P., Hamuro, Y., Raghuram, S., Wang, Y., Burris, T. P. \& Rastinejad, F. (2008). Nature, 456, 350-356.

Dubois, V., Eeckhoute, J., Lefebvre, P. \& Staels, B. (2017). J. Clin. Invest. 127, 1202-1214.

Emsley, P., Lohkamp, B., Scott, W. G. \& Cowtan, K. (2010). Acta Cryst. D66, 486-501.

Evans, P. R. \& Murshudov, G. N. (2013). Acta Cryst. D69, 1204-1214. Georgiadi, A. \& Kersten, S. (2012). Adv. Nutr. 3, 127-134.

Kabsch, W. (2010). Acta Cryst. D66, 125-132.

Kasuga, J. I., Ishida, S., Yamasaki, D., Makishima, M., Doi, T., Hashimoto, Y. \& Miyachi, H. (2009). Bioorg. Med. Chem. Lett. 19, 6595-6599.

Kasuga, J. I., Ishikawa, M., Yonehara, M., Makishima, M., Hashimoto, Y. \& Miyachi, H. (2010). Bioorg. Med. Chem. 18, 7164-7173.
Kasuga, J. I., Nakagome, I., Aoyama, A., Sako, K., Ishizawa, M., Ogura, M., Makishima, M., Hirono, S., Hashimoto, Y. \& Miyachi, H. (2007). Bioorg. Med. Chem. 15, 5177-5190.

Kasuga, J. I., Oyama, T., Nakagome, I., Aoyama, A., Sako, K., Makishima, M., Hirono, S., Morikawa, K., Hashimoto, Y. \& Miyachi, H. (2009). Yakugaku Zasshi, 129, 709-718.

Liebschner, D., Afonine, P. V., Baker, M. L., Bunkóczi, G., Chen, V. B., Croll, T. I., Hintze, B., Hung, L.-W., Jain, S., McCoy, A. J., Moriarty, N. W., Oeffner, R. D., Poon, B. K., Prisant, M. G., Read, R. J., Richardson, J. S., Richardson, D. C., Sammito, M. D., Sobolev, O. V., Stockwell, D. H., Terwilliger, T. C., Urzhumtsev, A. G., Videau, L. L., Williams, C. J. \& Adams, P. D. (2019). Acta Cryst. D75, 861-877.

Mirza, A. Z., Althagafi, I. I. \& Shamshad, H. (2019). Eur. J. Med. Chem. 166, 502-513.

Miyachi, H. (2021). Int. J. Mol. Sci. 22, 9223.

Müller, R. (2017). Biochimie, 136, 90-99.

Oyama, T., Kamata, S., Ishii, I. \& Miyachi, H. (2021). Biol. Pharm. Bull. 44, 1202-1209.

Oyama, T., Toyota, K., Waku, T., Hirakawa, Y., Nagasawa, N., Kasuga, J., Hashimoto, Y., Miyachi, H. \& Morikawa, K. (2009). Acta Cryst. D65, 786-795.

Strosznajder, A. K., Wójtowicz, S., Jeżyna, M. J., Sun, G. Y. \& Strosznajder, J. B. (2021). Neuromol Med. 23, 86-98.

Wu, C.-C., Baiga, T. J., Downes, M., La Clair, J. J., Atkins, A. R., Richard, S. B., Fan, W., Stockley-Noel, T. A., Bowman, M. E., Noel, J.-P. \& Evans, R. M. (2017). Proc. Natl Acad. Sci. USA, 114, E2563E2570.

Xu, H. E., Lambert, M. H., Montana, V. G., Parks, D. J., Blanchard, S. G., Brown, P. J., Sternbach, D. D., Lehmann, J. M., Wisely, G. B., Willson, T. M., Kliewer, S. A. \& Milburn, M. V. (1999). Mol. Cell, 3, 397-403.

Zarei, M., Aguilar-Recarte, D., Palomer, X. \& Vázquez-Carrera, M. (2021). Metabolism, 114, 154342. 